\title{
Differentiation of tumour-stage mycosis fungoides, psoriasis vulgaris and normal controls in a pilot study using serum proteomic analysis
}

\author{
E.W. Cowen, C-W. Liu, ${ }^{\star}$ S.M. Steinberg, $†$ S. Kang, $\$$ E.C. Vonderheid,§ H.S. Kwak, $\uparrow$ S. Booher, E.F. Petricoin, \\ L.A. Liotta, G. Whiteley* and S.T. Hwang \\ Dermatology Branch, Center for Cancer Research, National Cancer Institute, National Institutes of Health, Bethesda, MD 20892, U.S.A. \\ *SAIC-Frederick, Inc., Clinical Proteomics Reference Laboratory, Gaithersburg, MD, U.S.A. \\ $\dagger$ Biostatistics and Data Management Section, Center for Cancer Research, National Cancer Institute, Rockville, MD, U.S.A. \\ \$Department of Dermatology, University of Michigan Medical School, Ann Arbor, MI, U.S.A. \\ §Departments of Dermatology and Oncology, Johns Hopkins Medical Institutes, Baltimore, MD, U.S.A. \\ - Center for Applied Proteomics and Molecular Medicine, Department of Molecular and Microbiology, George Mason University, Manassas, VA, U.S.A.
}

\section{Summary}

\section{Correspondence \\ Edward W. Cowen. \\ E-mail: cowene@mail.nih.gov}

\section{Accepted for publication \\ 24 May 2007}

\section{Key words}

mycosis fungoides, proteomics, psoriasis

\section{Conflicts of interest}

None declared.
Background Serum proteomic analysis is an analytical technique utilizing highthroughput mass spectrometry (MS) in order to assay thousands of serum proteins simultaneously. The resultant 'proteomic signature' has been used to differentiate benign and malignant diseases, enable disease prognosis, and monitor response to therapy.

Objectives This pilot study was designed to determine if serum protein patterns could be used to distinguish patients with tumour-stage mycosis fungoides (MF) from patients with a benign inflammatory skin condition (psoriasis) and/or subjects with healthy skin.

Methods Serum was analysed from 45 patients with tumour-stage MF, 56 patients with psoriasis, and 47 controls using two MS platforms of differing resolution. An artificial intelligence-based classification model was constructed to predict the presence of the disease state based on the serum proteomic signature.

Results Based on data from an independent testing set (14-16 subjects in each group), MF was distinguished from psoriasis with $78.6 \%$ (or $78.6 \%$ ) sensitivity and $86.7 \%$ (or $93.8 \%$ ) specificity, while sera from patients with psoriasis were distinguished from those of nonaffected controls with $86.7 \%$ (or 93.8\%) sensitivity and $75.0 \%$ (or $76.9 \%$ ) specificity (depending on the MS platform used). MF was distinguished from unaffected controls with $61.5 \%$ (or $71.4 \%$ ) sensitivity and $91 \cdot 7 \%$ (or $92.9 \%$ ) specificity. In addition, a secondary survival analysis using 11 MS peaks identified significant survival differences between two MF groups (all P-values $<0 \cdot 05$ ).

Conclusions Serum proteomics should be further investigated for its potential to identify patients with neoplastic skin disease and its ability to determine disease prognosis.
Serum proteomics, the study of a subset of circulating proteins, is a novel method to study complex human disease such as cancer. Over the last several years, several reports have described the use of proteomic pattern analysis to determine new biomarkers of disease, ${ }^{1,2}$ enable disease prognosis ${ }^{3,4}$ and monitor response to therapy. ${ }^{5}$ Human skin is continuously perfused by blood, and its cellular components are bathed in extracellular fluid. It is therefore likely that proteins associated with neoplastic or inflammatory processes in the skin are released into the extracellular space, entering the lymphatic vessels, and, ultimately, the blood vascular system via the thoracic duct. Endothelial cells in skin also produce a variety of cytokines, chemokines and adhesion molecules that are released directly into the circulation during inflammatory skin reactions. Both of these processes may be expected to lead to quantitative as well as qualitative changes in the serum protein 
profile. Therefore, the detection of low molecular weight serum proteins by mass spectrometry (MS) and subsequent pattern analysis using software algorithms (serum proteomics) represents a potentially important method by which to diagnose and study skin disease.

Mycosis fungoides (MF) is responsible for nearly one-half of all primary cutaneous lymphomas as classified by the World Health Organization-European Organization for Research and Treatment of Cancer. ${ }^{6}$ It is characterized clinically by variable skin involvement, including patch, plaque and tumour-stage disease and rarely visceral involvement. Overt lymph node involvement occurs in about $20 \%$ and $50 \%$ of patients with plaque and tumour-stage $\mathrm{MF}$, respectively, ${ }^{7}$ and circulating neoplastic cells can be detected with sensitive methods even in patients with limited disease. ${ }^{8,9}$ At its onset, MF can be difficult to diagnose and the clinical manifestations may resemble benign dermatitis. ${ }^{10}$ The TNMB classification of MF recognizes four skin $\mathrm{T}$ ratings (T1, patches or plaques involving $<10 \%$ of skin; T2, patches or plaques involving $>10 \%$ of skin; $\mathrm{T} 3$, tumour(s); T4, erythroderma). ${ }^{11}$ While most patients with T1 disease can expect normal life expectancy, T2-T4-stage disease is associated with a progressive and dramatic decrease in life expectancy. ${ }^{12}$

In contrast, psoriasis is a relatively common benign inflammatory skin disease ${ }^{13}$ and appears to be a $\mathrm{T}$ cell-mediated response to putative autoantigen(s), leading to cytokine and chemokine activation in target tissue. ${ }^{14}$ A diagnosis of psoriasis can usually be made based on the clinical examination alone, and a skin biopsy is not routinely necessary. However, both psoriasis and early-stage MF are characterized by scaly, erythematous plaques.

In this pilot study, we sought to determine if serum protein patterns could potentially be used to differentiate patients with tumour-stage (T3) MF from patients with psoriasis and patients without inflammatory or neoplastic skin disease.

\section{Materials and methods}

\section{Patient selection}

Participants with tumour-stage (T3 skin rating) MF, moderateto-severe psoriasis, and healthy volunteers were eligible for entry into the study. MF patients with tumour-stage disease with histological confirmation were eligible to participate. Patients with psoriatic disease were required to demonstrate moderate-to-severe involvement (Psoriasis Area and Severity Index score of $\geq 12),{ }^{15}$ in order to maximize the likelihood that a distinct proteomic pattern might be identified for this disease. Criteria for exclusion from either group were systemic treatment for the skin disease 2 weeks prior to study entry or improvement in cutaneous disease over the last 2 weeks. Subjects were excluded if they had a known history of human immunodeficiency virus or human T-cell lymphotropic virus type 1 infection, or were pregnant.

Healthy volunteers were chosen based on an approximated mean age and gender ratio of the two disease groups (MF and psoriasis). Healthy volunteers were recruited from posted advertisements at the National Institutes of Health (NIH) Clinical Center and underwent a history and dermatological examination (E.W.C. or S.T.H.) to confirm the absence of significant inflammatory or neoplastic skin disease.

Patients with tumour-stage MF were identified from protocols for studying the disease at the NIH Clinical Center. Because of the rarity of the disease, additional stored serum samples from patients diagnosed with tumour-stage MF were collected from one of the authors (E.C.V.). Sera obtained from Johns Hopkins University (JHU) had been stored for 313 years (median 8). Patients with psoriasis were recruited through local advertisements at the NIH Clinical Center and via collaboration with an investigator at the University of Michigan (UM) (S.K.).

The protocol was reviewed by the Institutional Review Board (IRB) of the National Cancer Institute, as well as the IRBs of the collaborating institutions. Signed informed consent was obtained from all participants donating serum solely for the purpose of the study. Waivers of informed consent were obtained from IRBs for archived specimens. In order to preserve patient confidentiality, samples were coded with identifiers that did not link the identity of the patients to the serum samples or to clinical data.

\section{Specimen collection}

Venous blood $(20 \mathrm{~mL})$ in serum separator tubes was collected from each patient or volunteer using standard phlebotomy technique. The clotted blood was centrifuged within $2 \mathrm{~h}$ of collection at $1800 \mathrm{~g}$ for $5 \mathrm{~min}$, transferred to a $1-\mathrm{mL}$ transfer/storage tube, and frozen at -40 to $-70{ }^{\circ} \mathrm{C}$. Archived frozen serum (1-2 mL per aliquot) from collaborating institutions was packaged on dry ice prior to shipping.

\section{Specimen processing: surface-enhanced laser desorption and ionization-time of flight analysis}

All samples were frozen at $-70{ }^{\circ} \mathrm{C}$ or below before processing in order to minimize potential sampling and handling variation. Aliquots of fresh frozen sera were sent to the SAIC Clinical Proteomics Reference Laboratory (CPRL). Samples were thawed and $10-\mu \mathrm{L}$ aliquots were obtained and used immediately for high-resolution surface-enhanced laser desorption and ionization-time of flight (SELDI-TOF) analysis.

Analyses were performed using optimized CPRL standard operating procedure. All steps were carried out in a temperature and humidity controlled laboratory using a Hamilton Star robotic processor (Hamilton Company, Reno, NV, U.S.A.) equipped with a Ciphergen ${ }^{\circledR}$ bioprocessor to hold the arrays. Ciphergen ${ }^{\circledR}$ Q10 arrays (Ciphergen, Fremont, CA, U.S.A.) were pretreated with two applications of $100 \mu \mathrm{L}$ of $100 \mathrm{mmol} \mathrm{L}^{-1}$ phosphate buffer ( $\mathrm{pH} 7 \cdot 0$ ) for $5 \mathrm{~min}$ each. The Q10 arrays were selected because of their ability to bind albumin, which has been shown to be a source of informative peptides. Patient samples were diluted $1: 10$ in 
$100 \mathrm{mmol} \mathrm{L}^{-1}$ phosphate buffer $(\mathrm{pH} 7 \cdot 0)$ and mixed by aspiration and dispensing. Samples $(20 \mu \mathrm{L}$ each) were then dispensed into the bioprocessor containing the arrays in duplicate wells and allowed to incubate at room temperature for $30 \mathrm{~min}$. The arrays were washed five times with $100 \mathrm{mmol} \mathrm{L}^{-1}$ phosphate buffer and then with deionized water. Liquid was removed and the arrays were air-dried for $10 \mathrm{~min}$. Two applications of $2 \mu \mathrm{L}$ of matrix $\left[10 \mathrm{mg} \mathrm{mL}^{-1}\right.$ sinnipinic acid in $50 \%$ acetonitrile/water with $0.5 \%$ trifluoroacetic acid (TFA)] were done at 15-min intervals. Arrays were dried for a minimum of $15 \mathrm{~min}$ and spectra were generated via a Ciphergen ${ }^{\circledR}$ P4000 mass spectrometer with the following settings: source, $25 \mathrm{kV}(+)$ ion; mass range, 0-50 kDa; focus mass, $9000 \mathrm{Da}$; matrix attenuation, $1000 \mathrm{Da}$; sampling rate, $800 \mathrm{MHz}$; calibration, external -ACTH (7-38) and bovine ubiquitin; acquisition method, SELDI quantitation; warming shots,@2100 nJ (not added); data shots, 10 @ 1600 nj; detector bias, $2987 \mathrm{~V}$; partitions, 1 of 4 (total of 530 laser shots per spot).

\section{Specimen processing: PrOTOF ${ }^{\mathrm{TM}}$}

High-resolution mass spectra were produced and gathered using the PerkinElmer ProXPRESSION biomarker enrichment kit (PerkinElmer, Boston, MA, U.S.A.) following the manufacturer's directions. Serum samples were clarified by centrifugation and diluted 1:5 with binding buffer. Wells of Cibachrome blue plates were washed four times with $200 \mu \mathrm{L}$ of binding buffer. Sample $(100 \mu \mathrm{L})$ was then added to each well of the Cibachrome blue plate and drawn slowly by vacuum through the plate. Plates were then washed an additional four times with binding buffer. Each well was then loaded with $200 \mu \mathrm{L}$ of elution buffer and a clean collection plate was placed in the manifold. The liquid was drawn through the plate and the eluate was collected.

A ZipPlate (Millipore, Newton, MA, U.S.A.) was prepared by washing three times with $150 \mu \mathrm{L}$ of $100 \%$ acetonitrile. The eluate from the blue plates was loaded on to the ZipPlate and the liquid was drawn through by vacuum. The ZipPlate was then centrifuged at $1200 \mathrm{~g}$ for $5 \mathrm{~min}$ to remove any remaining liquid. A disposable matrix-assisted laser desorption and ionization (MALDI) plate (PerkinElmer) was prepared by rinsing with $100 \%$ ethanol and rinsing with water. The plate was dried and placed into the ZipPlate manifold with the MALDI target holder. Peptides were eluted and directly spotted on the target plate using $3 \mu \mathrm{L}$ of matrix $\left(10 \mathrm{mg} \mathrm{mL}^{-1}\right.$ alpha cyano in 50\% acetonitrile/water with $0.5 \%$ TFA) using a gentle vacuum and the target was air dried. Spectra were generated from target plates using a PerkinElmer prOTOF $^{\mathrm{TM}}$ orthogonal mass spectrometer with the following settings: laser shots, 20; laser pattern, $1 \mathrm{~mm}$ rings $01+03+05,48$ spots (960 laser shots total for each sample); laser energy, $80 \%$; laser rate, $80 \mathrm{~Hz}$; declustering, $30.0 \mathrm{~V}$; cooling flow, $150 \mathrm{~mL} \mathrm{~min}{ }^{-1}$; focusing flow, $200 \mathrm{~mL} \mathrm{~min}^{-1}$; mass range, 700-12 000 Da; expected high mass, 12000 Da; acceleration voltage, $16 \mathrm{kV}(+)$ ion; detector, $2300 \mathrm{~V}$.

\section{Spectra preprocessing: surface-enhanced laser desorption and ionization-time of flight analysis}

In order to reduce the effect of the mass drift problem inherent in the Ciphergen ${ }^{\circledR}$ low-resolution instrument, we first used the maximum probability peak alignment algorithm developed in-house to align peaks across different samples by using six reference points with $\mathrm{m} / \mathrm{z}$ approximating 225.4, $4158 \cdot 4,4473 \cdot 7,6639 \cdot 2,7775 \cdot 7$ and $9433 \cdot 4$. Next, the intensities of each spectrum were scaled using an adjusted coefficient so that the total ion current (TIC) of the scaled spectrum equalled the average TIC of all the spectra in the study. Spectra with extreme low TICs, or with adjusted coefficients $>3$, were treated as outliers and excluded from further analysis procedures. Finally, the spectra were binned using a uniform window size of $25 \mathrm{Da}$ from 250 to $14500 \mathrm{Da}$ to reduce the number of data points from approximately 30000 to 571 per spectrum.

\section{Spectra preprocessing: PrOTOF $^{\mathrm{TM}}$}

Spectra from the PerkinElmer PrOTOF ${ }^{\mathrm{TM}}$ instrument are high resolution and carry no mass drift effect, eliminating the need for peak alignment. TIC, however, varies across different spectra. Therefore, we used the same intensity scaling and outlier detection methods as described above for the SELDI-TOF data. The spectra were then binned using a linear growing window size, and the data points per spectrum were reduced to 10483 after binning.

\section{Proteomics pattern analysis}

In order to reduce the variations caused by the instruments or sample preparation process, every sample was analysed in duplicate, giving two spectra per patient in this study. The spectra were randomly divided into training (70\%) and testing sets $(30 \%)$ so that duplicate spectra of the same sample were assigned to the same set. The classification models were built from the training set, using an integrated proteomics biomarker discovery system developed in the CPRL, and applied to the testing set, which was wholly independent of the training set. The $\mathrm{m} / \mathrm{z}$ values in the models are based on the binned and/or normalized data, and not the actual $\mathrm{m} / \mathrm{z}$ values from the raw mass spectra.

The proteomics biomarker discovery system of CPRL integrates various feature selection methods and three machine learning algorithms: Partial Least Square regression from SAS (SAS Institute, Inc., Cary, NC, U.S.A.), SVM from LIBSVM (Chih-Chung Chang and Chih-Jen Lin, LIBSVM: a library for support vector machines, 2001) and C5.0 decision tree from RuleQuest (St Ives, NSW, Australia). The classification result for a sample was determined using the majority vote of the six classification results from those three algorithms on the duplicate spectra of a sample. If there was a tie (three results for each class), the sample was classified as 'not determined'. In the process of building models from the training set, each 
of the above three algorithms was independently tuned and optimized using 10-fold cross-validation. That is, $10 \%$ of the samples in the training set were left out every time for evaluation of the model. Models were built from the $90 \%$ training samples and the model performance was calculated from the $10 \%$ of samples that were left out each time. This process was repeated 10 times so that every sample was left out once. Model parameters were adjusted in order to achieve the best average performances on the 10 left out small sets that were used for the final model.

\section{Statistical considerations}

The study was originally intended to enrol 47 subjects from each of the three distinct subject populations, for model development, and then an additional 47 from each population for model testing. These values were chosen in order to have $79 \%$ power to rule out $80 \%$ sensitivity or specificity in favour of $95 \%$ sensitivity or specificity, with $99 \%$ one-sided confidence. However, due to differing accrual patterns than projected, the study was refocused as a pilot study, and those results are reported herein, in which $70 \%$ of the data (31-40 subjects per group) would be used to develop the classifier and the remainder (14-16 subjects per group) to test the model. All results found in this study are considered preliminary and would require validation in much larger, independent groups of subjects before being considered worthwhile for use in the practice setting.

Age and gender differences between each set of two groups were examined using the Wilcoxon rank sum test and Fisher's exact test, respectively. The success of the model in differentiating each pair of groups based on the serum proteomic signatures was expressed in terms of sensitivity (true positives/true positives and false negatives) and specificity (true negatives/true negatives and false positives) and reported with an exact two-sided 95\% confidence interval.

As a secondary evaluation, survival analysis was performed on patients with MF based on the presence of specific ion peaks. The overall survival time was calculated from the date of specimen collection to the date of last contact or date of death. For the high-resolution prOTOF ${ }^{\mathrm{TM}}$ data, peaks were chosen based on their involvement in the classification process, if they had highly significant differences between healthy and MF samples based on a two-sided Wilcoxon rank sum test. Univariate Cox proportional hazard models were then used to determine whether a peak is associated with the probability of survival. Those peaks with P-values $<0.05$ were further used to categorize the patients with MF. Kaplan-Meier curves were generated based on dichotomizing patients according to their levels for the above-mentioned significant peaks. A log-rank test was used to determine the degree to which the patients in those low-expression and high-expression groups demonstrated a significant difference in survival probability. All survival analyses were performed using SAS software (version 9.1; SAS Institute, Inc.). All P-values are two-tailed and have not been adjusted for multiple comparisons.

\section{Results}

\section{Demographics of patient populations}

Demographic data for MF, psoriasis and healthy control groups are provided in Table 1. At the time of blood sampling, patients with MF were older than healthy controls (median 67 years vs. 50 years, respectively; $P<0.001$ ) and patients with psoriasis (median 45.5 years; $P<0.001$ ). In addition, MF patients evaluated at the NIH (median age 58 years) were younger at the time of sampling than patients from JHU (median age 70 years; $P=0.035$ ). There was no significant difference in age between the psoriasis and healthy control groups $(P=0 \cdot 19)$. In addition, there were no significant gender differences among the disease groups ( $P>0.05$ for all groups); however, the NIH-based psoriasis samples had significantly fewer females than the UM-based psoriasis samples $(P=0.027)$. There was no significant difference in age or gender between the model and test samples within each disease group ( $P>0.05$ for all groups).

\section{Proteomic analysis}

Comparisons were made between serum samples as follows: (i) MF patients vs. healthy controls; (ii) psoriasis patients vs. healthy controls; and (iii) MF patients vs. psoriasis patients (Table 2). Proteomic modelling successfully distinguished MF patient serum samples from samples from healthy controls and from patients with psoriasis with reasonable accuracy using the SELDI-TOF Ciphergen ${ }^{\circledR}$ MS-based data (Table 3). The sensitivity of detection of the disease state was highest in the psoriasis vs. healthy control comparison (93.75\%), while the specificity of this comparison was $75 \cdot 0 \%$. Although, by comparison, the sensitivity to detect MF was lower in both the MF vs. unaffected and MF vs. psoriasis groups, the specificity to detect MF in both models was $>90 \%$ (91.67\% and $93 \cdot 75 \%$, respectively).

Modelling based on the higher-resolution PrOTOF $^{\mathrm{TM}}$ data yielded equivalent sensitivity to the Ciphergen ${ }^{\circledR}$ MS-based data for the detection of MF vs. psoriasis patients; however, the sensitivity for detection of the MF disease state vs. unaffected samples increased using the PrOTOF ${ }^{\mathrm{TM}}$ data $(71 \cdot 43 \%$; Table 3$)$. As with the Ciphergen ${ }^{\circledR}$ MS-based data, the specificity to differentiate MF from unaffected samples and psoriasis samples approached or exceeded $90 \%$ in both comparison groups. In all cases, confidence intervals are fairly wide, indicating the potential variability in the results obtained.

It is theoretically possible that differences in handling, storage or other unknown factors among the three institutions participating in this study may have yielded proteomic signatures that contributed to the classification of normal and disease states. To assess this possibility, we sought to build a proteomic classification model that could distinguish psoriasis samples from the NIH and UM (the sources of psoriasis samples) and MF samples from the NIH and JHU (the sources of tumour-stage MF samples). Interestingly, no proteomic 
Table 1 Demographic characteristics of patient serum samples

\begin{tabular}{|c|c|c|c|c|c|c|}
\hline & $\mathrm{NIH}$ & $\mathrm{UM}$ & JHU & Model set & Test set & Total \\
\hline \multicolumn{7}{|l|}{ Tumour-stage MF } \\
\hline Number of samples (\%) & $14(31 \%)$ & & $31(69 \%)$ & $31(69 \%)$ & $14(31 \%)$ & $45(100 \%)$ \\
\hline Age, years (mean \pm SEM) & $56.64 \pm 3.99$ & & $66 \cdot 52 \pm 2.46$ & $63 \cdot 97 \pm 2.46$ & $62.29 \pm 4.58$ & $63 \cdot 44 \pm 2 \cdot 19$ \\
\hline Median age, years (range) & $58(30-77)$ & & $70(30-85)$ & $67(33-82)$ & $67 \cdot 5(30-85)$ & $67(30-85)$ \\
\hline Females & 2 & & 10 & 8 & 4 & 12 \\
\hline Males & 12 & & 21 & 23 & 10 & 33 \\
\hline Black & 4 & & 7 & 9 & 2 & 11 \\
\hline Caucasian & 9 & & 23 & 22 & 10 & 32 \\
\hline Other & 1 & & 1 & 0 & 2 & 2 \\
\hline \multicolumn{7}{|l|}{ Psoriasis } \\
\hline Number of samples (\%) & $8(14 \%)$ & $48(86 \%)$ & & $40(71 \%)$ & $16(29 \%)$ & $56(100 \%)$ \\
\hline Age, years (mean \pm SEM) & $51 \cdot 75 \pm 5 \cdot 44$ & $47 \cdot 27 \pm 1 \cdot 81$ & & $46 \cdot 63 \pm 1 \cdot 73$ & $51 \cdot 13 \pm 4 \cdot 22$ & $47 \cdot 91 \pm 1.73$ \\
\hline Median age, years (range) & $45(30-77)$ & $45(21-78)$ & & $44(28-68)$ & $49(21-78)$ & $45 \cdot 5(21-78)$ \\
\hline Females & 3 & 13 & & 10 & 6 & 16 \\
\hline Males & 5 & 35 & & 30 & 10 & 40 \\
\hline Black & 0 & 4 & & 2 & 2 & 4 \\
\hline Caucasian & 6 & 43 & & 35 & 14 & 49 \\
\hline Other & 2 & 1 & & 3 & 0 & 3 \\
\hline \multicolumn{7}{|l|}{ Healthy controls } \\
\hline Number of samples (\%) & $47(100 \%)$ & & & $33(70 \%)$ & $14(30 \%)$ & $47(100 \%)$ \\
\hline Age, years (mean \pm SEM) & $49 \cdot 72 \pm 1 \cdot 24$ & & & $48 \cdot 85 \pm 1 \cdot 44$ & $51.79 \pm 2.39$ & $49 \cdot 72 \pm 1 \cdot 24$ \\
\hline Median age, years (range) & $50(32-70)$ & & & $50(32-64)$ & $51 \cdot 5(33-70)$ & $50(32-70)$ \\
\hline Females & 22 & & & 14 & 8 & 22 \\
\hline Males & 25 & & & 19 & 6 & 25 \\
\hline Black & 6 & & & 5 & 1 & 6 \\
\hline Caucasian & 35 & & & 24 & 11 & 35 \\
\hline Other & 6 & & & 4 & 2 & 6 \\
\hline
\end{tabular}

MF, mycosis fungoides; NIH, National Institutes of Health Clinical Center; UM, University of Michigan; JHU, Johns Hopkins University.

Table 2 Comparison of predicted disease state with true state by serum proteomic analysis using Ciphergen-based and PrOTOF-based data

\begin{tabular}{|c|c|c|c|c|c|c|c|}
\hline \multicolumn{4}{|l|}{ Ciphergen } & \multicolumn{4}{|l|}{ PrOTOF } \\
\hline MF vs. unaffected & Predicted: MF & Predicted: unaffected & ND & MF vs. unaffected & Predicted: MF & Predicted: unaffected & ND \\
\hline True state: MF & 8 & 5 & 1 & True state: MF & 10 & 4 & \\
\hline True state: unaffected & 1 & 11 & 2 & True state: unaffected & 1 & 13 & \\
\hline Psoriasis vs. unaffected & Predicted: psoriasis & Predicted: unaffected & ND & Psoriasis vs. unaffected & Predicted: psoriasis & Predicted: unaffected & ND \\
\hline True state: psoriasis & 15 & 1 & & True state: psoriasis & 13 & 2 & 1 \\
\hline True state: unaffected & 3 & 9 & 2 & True state: unaffected & 3 & 10 & 1 \\
\hline MF vs. psoriasis & Predicted: MF & Predicted: psoriasis & ND & MF vs. psoriasis & Predicted: MF & Predicted: psoriasis & ND \\
\hline True state: MF & 11 & 3 & & True state: MF & 11 & 3 & \\
\hline True state: psoriasis & 1 & 15 & & True state: psoriasis & 2 & 13 & 1 \\
\hline
\end{tabular}

signature was identified that could distinguish between psoriasis samples from the NIH and the UM (data not shown). While a proteomic classification model was developed that differentiated MF samples from the NIH and those from JHU (data not shown), the peaks contained in this signature were distinct from the peaks used to distinguish normal and psoriatic patient sera from sera of patients with tumour-stage MF. Thus, while differences may exist between the signatures of sera from NIH and JHU based on institutional factors, these differences are apparently distinct from those that classify disease status.

\section{Survival analysis}

As a secondary evaluation, survival analysis was performed for the 45 patients with MF based on the high-resolution PrOTOF $^{\mathrm{TM}}$ data. Eleven peaks involved in the classification of healthy vs. MF samples were chosen. Differences between the two groups were highly statistically significant, with all Wilcoxon rank sum test $\mathrm{P}$-values $<0.0001$ (in fact, all had $\mathrm{P}<10^{-10}$ ). They also had clear visual spectra differences (plot not shown). Univariate Cox model evaluations indicated that these peaks were each associated with the probability of 
Table 3 Sensitivity and specificity of serum proteomic analysis with independent test groups (95\% two-sided confidence intervals)

\begin{tabular}{lll}
\hline & Sensitivity $(\%)$ & Specificity (\%) \\
\hline Ciphergen & & \\
MF $(\mathrm{n}=13)$ vs. unaffected $(\mathrm{n}=12)$ & $61 \cdot 54(31 \cdot 5-86 \cdot 1)$ & $91 \cdot 67(61 \cdot 9-99 \cdot 8)$ \\
MF $(\mathrm{n}=14)$ vs. psoriasis $(\mathrm{n}=16)$ & $78 \cdot 57(49 \cdot 2-95 \cdot 3)$ & $93 \cdot 75(69 \cdot 8-99 \cdot 8)$ \\
Psoriasis $(\mathrm{n}=16)$ vs. unaffected $(\mathrm{n}=12)$ & $93 \cdot 75(69 \cdot 8-99 \cdot 8)$ & $75 \cdot 0(42 \cdot 8-94 \cdot 5)$ \\
PrOTOF & & \\
MF $(\mathrm{n}=14)$ vs. unaffected $(\mathrm{n}=14)$ & $71 \cdot 43(41 \cdot 9-91 \cdot 6)$ & $92 \cdot 86(66 \cdot 1-99 \cdot 8)$ \\
MF $(\mathrm{n}=14)$ vs. psoriasis $(\mathrm{n}=15)$ & $78 \cdot 57(49 \cdot 2-95 \cdot 3)$ & $86 \cdot 67(59 \cdot 5-98 \cdot 3)$ \\
Psoriasis $(\mathrm{n}=15)$ vs. unaffected $(\mathrm{n}=13)$ & $86 \cdot 67(59 \cdot 5-98 \cdot 3)$ & $76 \cdot 92(46 \cdot 2-95 \cdot 0)$ \\
\hline MF, mycosis fungoides. & & \\
\hline
\end{tabular}

Table 4 Peaks with Wilcoxon rank sum P-values $<0.0001$ for the difference between healthy and mycosis fungoides (MF) samples, and strongly associated with MF patient survival

\begin{tabular}{|c|c|c|c|}
\hline \multirow[b]{2}{*}{ Peak } & \multicolumn{3}{|c|}{$\begin{array}{l}\text { Univariate Cox proportional hazard model } \\
\text { results }\end{array}$} \\
\hline & $\begin{array}{l}\text { Likelihood } \\
\text { ratio test } \\
\text { P-value }\end{array}$ & $\begin{array}{l}\text { Score test } \\
\text { P-value }\end{array}$ & $\begin{array}{l}\text { Wald test } \\
\text { P-value }\end{array}$ \\
\hline Mz_2080_6 & $<0.0001$ & $<0.0001$ & $<0.0001$ \\
\hline $\mathrm{Mz}$ 1076_3 & 0.026 & 0.033 & 0.040 \\
\hline $\mathrm{Mz} \_2208 \_6$ & 0.0001 & $<0.0001$ & $<0.0001$ \\
\hline $\mathrm{Mz}$-3158_1 & 0.0001 & $<0.0001$ & $<0.0001$ \\
\hline Mz_1944_4 & $<0.0001$ & $<0.0001$ & 0.0001 \\
\hline Mz_3523_2 & $0 \cdot 0001$ & $<0.0001$ & $<0.0001$ \\
\hline $\mathrm{Mz}$-3521_3 & 0.0001 & $<0.0001$ & 0.0001 \\
\hline Mz_3526_3 & 0.0001 & $<0.0001$ & 0.0001 \\
\hline $\mathrm{Mz} \_3683 \_4$ & 0.0002 & $<0.0001$ & 0.0001 \\
\hline $\mathrm{Mz}$-3543_3 & 0.0035 & 0.0020 & 0.0033 \\
\hline $\mathrm{Mz} \_3770 \_3$ & 0.0004 & $<0.0001$ & 0.0002 \\
\hline
\end{tabular}

survival in this small set of patients $(P<0.05$; see Table 4$)$. Those 11 peaks were further used to categorize the 45 patients with MF into those with below mean peak intensity and those with above mean peak intensity. All the peaks, except peaks 1076 and 3543, had log-rank test P-value <0.05 when dichotomized in this fashion. In a limited set of patients, this indicates that MF patients with higher peak expression have significantly different survival probabilities compared with MF patients with lower peak expression. The Kaplan-Meier curves for two illustrative peaks are presented in Figure 1.

\section{Discussion}

In this study, we present the first serum proteomic analysis of tumour-stage MF and describe the potential usefulness of serum proteomic analysis to differentiate patients with tumourstage MF from patients with psoriasis as well as unaffected controls with relatively good sensitivity and specificity.

The study design described herein uses a conservative approach to determine the presence of valid proteomic signa- tures. Dividing the initial samples into independent training and testing (validation) sets diminishes the test group sample size and, therefore, also diminishes the power to detect important differences between the groups, but this method avoids the pitfall of model over-fitting using cross-validation techniques (using the same sample for both testing and training). Despite this restriction, differences were found in our test groups based on models constructed from a relatively small number of MF and psoriasis samples. Additionally, data were modelled from two independent MS analyses, both of which yielded similar results for each disease comparison. The higher-resolution capabilities of the PrOTOF ${ }^{\mathrm{TM}}$ unit led to disease discrimination that was similar to the lower-resolution machine in each comparison group.

There are several limitations to this study. The ages reported in each group correspond to the patient age at the time of blood sampling, not at the time of initial diagnosis. Because the healthy volunteer group was used for comparison with both MF and psoriasis, an attempt was made to approximate a mean age between the two disease groups when healthy volunteers were initially recruited. Nevertheless, a significant difference in age was identified between patients with MF and the other two groups, which may potentially have led to pattern discrimination based on age rather than disease state. There may be other undetected heterogeneity between the groups that would further confound our results.

Lack of reproducibility has been a valid criticism of proteomic analysis. ${ }^{16}$ Because the samples were unlinked from the patients after they were drawn, we are not able to test the reproducibility of our results by multiple assays over time in our patients. However, the protocol and environmental conditions for reproducibility have been defined by the CPRL, and controls tested alongside the samples indicated that the technology would not likely be a significant source of error.

By contrast, the method described herein was able to predict the disease state of many of the patients accurately despite the inherent heterogeneity within the classification groups. Although other cutaneous diseases were excluded from the protocol, patients may have had other concurrent systemic disease processes that share similar inflammatory or neoplastic signatures with psoriasis and MF. Heterogeneity within each disease category is also possible. For instance, Carlen et al. 


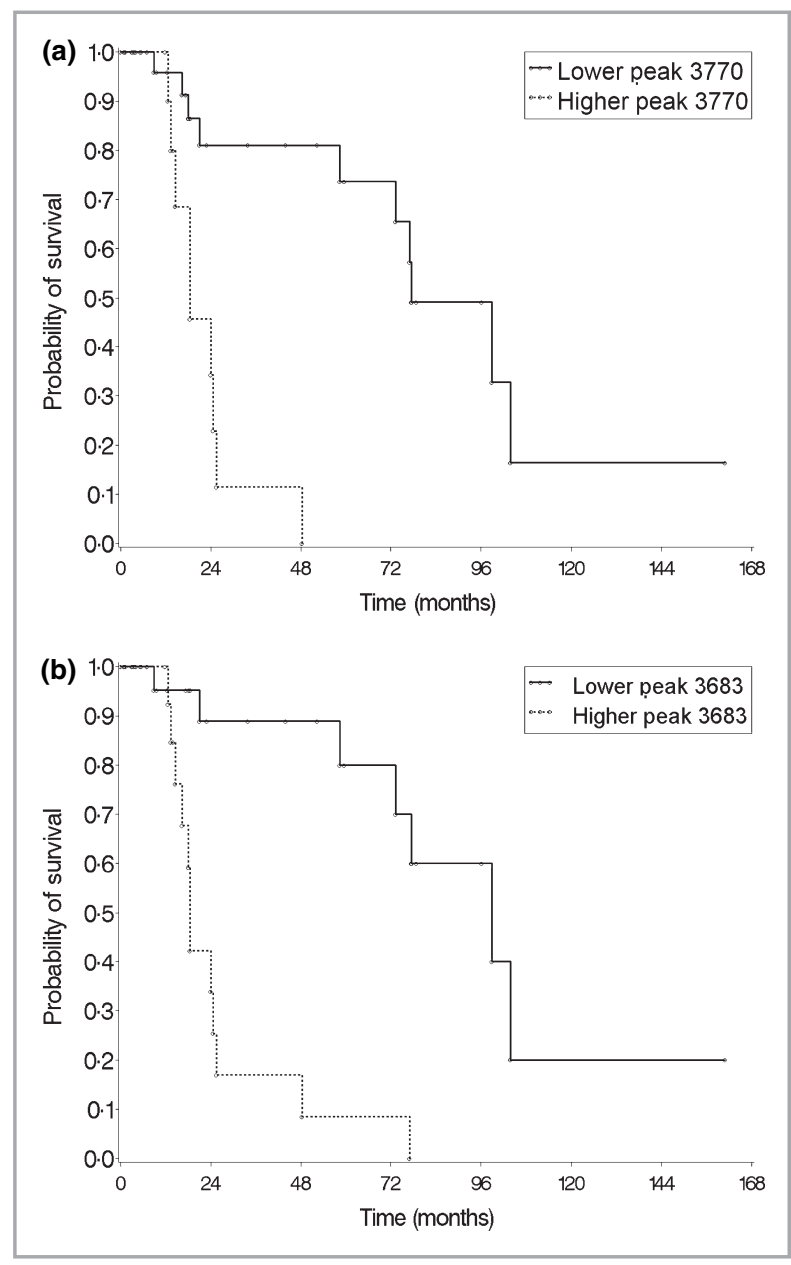

Fig 1. (a) Kaplan-Meier curves for patients with higher vs. lower peak 3770. (b) Kaplan-Meier curves for patients with higher vs. lower peak 3683.

recently reported protein expression differences between acute guttate and chronic plaque psoriasis. ${ }^{17}$

Patients with psoriasis were chosen as a comparison group to $\mathrm{MF}$ in this study because their aetiologies are thought to be distinct. Both are $\mathrm{T}$ cell-mediated inflammatory disorders, but the neoplastic cells in MF are polarized to release Th2 cytokines whereas psoriasis has a Th1 profile. As this was a pilot 'proof of concept' study, patients with tumour-stage MF were considered most likely of the primary cutaneous MF (non-Sézary) patients to possess a distinct serum proteomic signature. If proteomic analysis did not identify distinctive proteomic differences between tumour-stage MF and psoriasis, it would be unlikely that it could be used to distinguish patch (or plaque) stage MF and psoriasis. It is possible that the differentiation we observed was due to a unique signature of advanced (tumour-stage) MF, and that patch and plaque-stage MF disease would not be as readily discernable from nonneoplastic inflammatory disease. For this reason, comparison between different stages of MF is an important next step in confirming the value of serum proteomic pattern analysis for the study of cutaneous lymphoma. Further study of MF at different stages of disease is necessary to determine if a unique signature is present in other stages of disease and to determine whether this approach may be useful for prognostication of the small percentage of patients who will have significant disease progression.

Proteomic analysis allows for high-throughput screening of thousands of proteins, and is potentially a powerful tool to identify multiple disease markers using an unbiased approach. Protein expression patterns have been shown to distinguish lymphoid neoplasm cell lines, ${ }^{18}$ identify potential tumour markers in anaplastic lymphoma cell lines ${ }^{19}$ and differentiate benign and malignant B-cell neoplastic tissue specimens. ${ }^{20}$ It was not possible to determine if the source of the differences between the circulatory proteome of patients with MF and subjects without MF in this study was due to the neoplastic cells or to a systemic response to the tumours.

The complexity of the serum proteome and the limitations of current protein technology present a significant challenge for definitive identification of peptides and protein fragments. However, new procedures have been proposed which successfully combine the advantages of high-throughput protein pattern analysis using high-resolution MS for the identification of protein biomarkers. ${ }^{21}$ Biomarker identification in MF could potentially provide important insight into the biology of tumour-stage MF in patients who had poorer survival. In addition, the visibility and relatively easy access to affected tissue in cutaneous diseases will allow for potential correlation, validation and reproducibility studies combining serum and tissue proteomic techniques.

In conclusion, we show that serum proteomic signatures are potentially able to differentiate patients with tumour-stage MF from unaffected controls as well as patients with psoriasis, and may enable prognosis among patients with tumour-stage disease. These preliminary findings merit further investigation and confirmation with larger cohorts of patients in order to define more precisely the classification ability of serum proteomic analysis. Although most patients with MF will eventually die of non-MF-related causes, a significant percentage of patients, particularly patients with tumour-stage disease, will have a progressive course resulting in significant morbidity and mortality. ${ }^{12}$ Proteomic analysis may allow for a better understanding of the heterogeneity in clinical disease observed with MF and potentially may provide important prognostic information.

\section{Acknowledgments}

This research was supported in part by the Intramural Research Program of the NIH, National Cancer Institute, Center for Cancer Research.

\section{References}

1 Petricoin EF, Ardekani AM, Hitt BA et al. Use of proteomic patterns in serum to identify ovarian cancer. Lancet 2002; 359:572-7. 
2 Simpkins F, Czechowicz JA, Liotta L et al. SELDI-TOF mass spectrometry for cancer biomarker discovery and serum proteomic diagnostics. Pharmacogenomics 2005; 6:647-53.

3 Ricolleau G, Charbonnel C, Lode L et al. Surface-enhanced laser desorption/ionization time of flight mass spectrometry protein profiling identifies ubiquitin and ferritin light chain as prognostic biomarkers in node-negative breast cancer tumors. Proteomics 2006; 6:1963-75.

4 Albitar M, Potts SJ, Giles FJ et al. Proteomic-based prediction of clinical behavior in adult acute lymphoblastic leukemia. Cancer 2006; 106:1587-94.

5 Espina V, Dettloff KA, Cowherd S et al. Use of proteomic analysis to monitor responses to biological therapies. Expert Opin Biol Ther 2004; 4:83-93.

6 Willemze R, Jaffe ES, Burg G et al. WHO-EORTC classification for cutaneous lymphomas. Blood 2005; 105:3768-85.

7 Vonderheid EC, Diamond LW, Lai SM et al. Lymph node histopathologic findings in cutaneous T-cell lymphoma. A prognostic classification system based on morphologic assessment. Am J Clin Pathol 1992; 97:121-9.

8 Fraser-Andrews EA, Woolford AJ, Russell-Jones R et al. Detection of a peripheral blood $\mathrm{T}$ cell clone is an independent prognostic marker in mycosis fungoides. J Invest Dermatol 2000; 114:117-21.

9 Muche JM, Lukowsky A, Asadullah K et al. Demonstration of frequent occurrence of clonal $\mathrm{T}$ cells in the peripheral blood of patients with primary cutaneous T-cell lymphoma. Blood 1997; 90: $1636-42$.

10 Zackheim HS, McCalmont TH. Mycosis fungoides: the great imitator. J Am Acad Dermatol 2002; 47:914-18.
11 Bunn PA Jr, Lamberg SI. Report of the committee on staging and classification of cutaneous T-cell lymphomas. Cancer Treat Rep 1979; 63:725-8

12 Zackheim HS, Amin S, Kashani-Sabet M et al. Prognosis in cutaneous T-cell lymphoma by skin stage: long-term survival in 489 patients. J Am Acad Dermatol 1999; 40:418-25.

13 Koo JY. Current consensus and update on psoriasis therapy: a perspective from the U.S. J Dermatol 1999; 26:723-33.

14 Schon MP, Boehncke WH. Psoriasis. N Engl J Med 2005; 352:1899912.

15 Fredriksson T, Pettersson U. Severe psoriasis - oral therapy with a new retinoid. Dermatologica 1978; 157:238-44.

16 Diamandis EP. Analysis of serum proteomic patterns for early cancer diagnosis: drawing attention to potential problems. J Natl Cancer Inst 2004; 96:353-6.

17 Carlen LM, Sanchez F, Bergman AC et al. Proteome analysis of skin distinguishes acute guttate from chronic plaque psoriasis. J Invest Dermatol 2005; 124:63-9.

18 Fujii K, Kondo T, Yokoo H et al. Protein expression pattern distinguishes different lymphoid neoplasms. Proteomics 2005; 5:4274-86.

19 Cussac D, Pichereaux C, Colomba A et al. Proteomic analysis of anaplastic lymphoma cell lines: identification of potential tumour markers. Proteomics 2006; 6:3210-22.

20 Fan G, Molstad M, Braziel RM et al. Proteomic profiling of mature CD10+ B-cell lymphomas. Am J Clin Pathol 2005; 124:920-9.

21 Lopez Z, Mikulskis A, Kuzdzal S et al. A novel, high-throughput workflow for discovery and identification of serum carrier proteinbound peptide biomarker candidates in ovarian cancer samples. Clin Chem 2007; 53:1067-74. 